\title{
Expansion of a Fermi cloud in the BCS-BEC crossover
}

\author{
G. Diana, ${ }^{1}$ N. Manini, ${ }^{1}$ and L. Salasnich ${ }^{1,2}$ \\ ${ }^{1}$ Dipartimento di Fisica and CNR-INFM, Università di Milano, Via Celoria 16, 20133 Milano, Italy \\ ${ }^{2}$ CNR-INFM and CNISM, Unità di Milano, Via Celoria 16, 20133 Milano, Italy
}

(Received 1 March 2006; published 6 June 2006)

\begin{abstract}
We study the free expansion of a dilute two-component Fermi gas with attractive interspecies interaction in the Bardeen-Cooper-Schrieffer-Bose-Einstein condensate (BCS-BEC) crossover. We apply a time-dependent parameter-free density-functional theory by using two choices of the equation of state: an analytic formula based on Monte Carlo data and the mean-field equation of state resulting from the extended BCS equations. The calculated axial and transverse radii and the aspect ratio of the expanding cloud are compared to experimental data on vapors of ${ }^{6} \mathrm{Li}$ atoms. Remarkably, the mean-field theory shows a better agreement with the experiments than the theory based on the Monte Carlo equation of state. Both theories predict a measurable dependence of the aspect ratio on expansion time and on scattering length.
\end{abstract}

DOI: 10.1103/PhysRevA.73.065601

PACS number(s): 03.75.Kk

\section{INTRODUCTION}

Current experiments with cold vapors of ${ }^{6} \mathrm{Li}$ and ${ }^{40} \mathrm{~K}$ atoms can operate in the regime of deep Fermi degeneracy. The available experimental data on two-hyperfinecomponent Fermi gases are concentrated across a Feshbach resonance, where the $s$-wave scattering length $a_{F}$ of the interatomic potential varies from large negative to large positive values $[1-4]$ and where a crossover from a BardeenCooper-Schrieffer (BCS) superfluid to a Bose-Einstein condensate (BEC) of molecular pairs has been predicted [5-7]. In these experiments, the Fermi cloud is dilute because the effective range $R_{0}$ of the interaction is much smaller than the mean interparticle distance, i.e., $k_{F} R_{0} \ll 1$, where $k_{F}=\left(3 \pi^{2} n\right)^{1 / 3}$ is the Fermi wave vector and $n$ is the gas number density. Even in this dilute regime the $s$-wave scattering length $a_{F}$ can be made very large: the interaction parameter $k_{F} a_{F}$ diverges and changes sign at a Feshbach resonance, despite $k_{F} R_{0}$ remaining small [1-3,8].

Recent experimental and theoretical investigations studied the density profiles [9-11], collective excitations $[9,10,12-17]$, condensate fraction [18-21] and vortices $[22,23]$ of the fermion cloud through the BCS-BEC crossover. In this Brief Report we analyze the free expansion of the Fermi gas through this crossover by using a parameterfree time-dependent density-functional theory $[16,17]$ based on the bulk equation of state of the superfluid, and including a quantum-pressure term. We adopt two possible equations of state: a reliable analytical interpolating formula based on bulk Monte Carlo results [17] and the mean-field equation of state based on extended BCS equations [5-7,14]. Experimentally, the free expansion of superfluid ${ }^{6} \mathrm{Li}$ clouds was observed by O'Hara et al. [1] and by Bourdel et al. [4]. The comparison of our theory with these experimental data shows that the effects of interaction could be detected during the expansion if the thermal component was negligible. In addition, by using local scaling equations, we investigate the long-time dynamics of the Fermi gas predicting novel and measurable effects of interaction on the time evolution of the expansion process.

\section{THEORY}

To describe the dynamics of a zero-temperature Fermi cloud in the external potential $U(\mathbf{r}, t)$ we use a hydrodynamic model with a von Weizsäcker quantum-pressure term. This theoretical approach is expected to be reliable for studying the collective dynamics of the Fermi gas $[16,17]$. The action functional $A[\psi]$ of the theory depends on the superfluid order parameter $\psi(\mathbf{r}, t)$ as follows:

$$
A=\int d t d^{3} \mathbf{r} \mathcal{L}\left(\psi, \partial_{t} \psi, \nabla \psi\right)
$$

where the Lagrangian density reads

$$
\mathcal{L}=i \hbar \psi^{*} \partial_{t} \psi+\frac{\hbar^{2}}{2 m} \psi^{*} \nabla^{2} \psi-U|\psi|^{2}-\mathcal{E}\left(|\psi|^{2}\right)|\psi|^{2} .
$$

$\mathcal{E}$ represents the bulk energy per particle of the system, which is conveniently expressed as a function of the number density $n=|\psi|^{2}$ by the following equation:

$$
\mathcal{E}(n)=\frac{3}{5} \frac{\hbar^{2} k_{F}^{2}}{2 m} f(y),
$$

where $f(y)$ is a function of the inverse interaction parameter $y=\left(k_{F} a_{F}\right)^{-1}$. In the weakly attractive regime $(y \ll-1)$ one expects a BCS Fermi gas of weakly bound Cooper pairs where the superfluid gap energy $\Delta$ is exponentially small. In the so-called unitarity limit $(y=0)$ one expects that the energy per particle is proportional to that of a noninteracting Fermi gas with a $n$-independent coefficient $f(0)=0.42$ [24]. In the weak-coupling BEC regime $(y \gg 1)$, a weakly repulsive Bose gas of dimers of mass $m_{B}=2 m$ and density $n_{B}=n / 2$ is expected. Such Bose-condensed molecules interact with a positive scattering length $a_{B}=0.6 a_{F}[25,26]$. The function $f(y)$ is modeled by the analytical formula

$$
f(y)=\alpha_{1}-\alpha_{2} \arctan \left(\alpha_{3} y \frac{\beta_{1}+|y|}{\beta_{2}+|y|}\right)
$$

recently derived [17] from Monte Carlo (MC) simulations $[26,27]$ and asymptotic expressions. Table 1 of Ref. [17] re- 
ports the values of the interpolating $\alpha_{1}, \alpha_{2}, \alpha_{3}, \beta_{1}$, and $\beta_{2}$.

In the present investigation we take an axially symmetric harmonic potential as a confining trap

$$
U(\mathbf{r}, t)=\frac{m}{2}\left[\bar{\omega}_{\rho}(t)^{2}\left(x^{2}+y^{2}\right)+\bar{\omega}_{z}(t)^{2} z^{2}\right],
$$

where $\bar{\omega}_{j}(t)=\omega_{j} \Theta(-t)$, with $j=1,2,3=\rho, \rho, z$ and $\Theta(t)$ is the step function, so that, after the external trap is switched off at $t>0$, the Fermi cloud performs a free expansion. The EulerLagrange equation for the field $\psi(\mathbf{r}, t)$ is obtained by minimizing the action functional of Eqs. (1) and (2). This leads to a time-dependent nonlinear Schrödinger equation (TDNLSE)

$$
i \hbar \partial_{t} \psi=\left[-\frac{\hbar^{2}}{2 m} \nabla^{2}+U+\mu\left(|\psi|^{2}\right)\right] \psi
$$

The nonlinear term $\mu$ is the bulk chemical potential of the system. Like the energy $\mathcal{E}$ of Eq. (3), also the bulk chemical potential $\mu$ is a function of the number density $n$. The MC chemical potential is related to the MC energy by the thermodynamical formula

$$
\mu(n)=\frac{\partial(n \mathcal{E}(n))}{\partial n}=\frac{\hbar^{2} k_{F}^{2}}{2 m}\left[f(y)-\frac{y}{5} f^{\prime}(y)\right] .
$$

Instead of the MC equation of state (7) based on Eq. (4), in the TDNLSE (6) one can plug the mean-field equation of state, obtained from the extended BCS (EBCS) equations [5-7]. In this scheme, the chemical potential $\mu$ and the gap energy $\Delta$ of the uniform Fermi gas are found by solving the following EBCS equations:

$$
\begin{gathered}
-\frac{1}{a_{F}}=\frac{2(2 m)^{1 / 2}}{\pi \hbar^{3}} \Delta^{1 / 2} I_{1}\left(\frac{\mu}{\Delta}\right), \\
n=\frac{(2 m)^{3 / 2}}{2 \pi^{2} \hbar^{3}} \Delta^{3 / 2} I_{2}\left(\frac{\mu}{\Delta}\right),
\end{gathered}
$$

where $I_{1}(x)$ and $I_{2}(x)$ are two monotonic functions which can be expressed in terms of elliptic integrals [20,28]. By solving these two EBCS equations we obtain the chemical potential $\mu$ as a function of $n$ and $a_{F}$, which can be inserted into the TDNLSE (6).

\section{EXPANSION OF FERMI GAS AND SCALING EQUATIONS}

The free expansion of a droplet of $1.5 \times 10^{5}{ }^{6} \mathrm{Li}$ atoms in the unitarity limit $(y \simeq 0)$ was investigated experimentally in Ref. [1]. The harmonic potential is anisotropic with $\lambda$ $=\omega_{z} / \omega_{\rho}=0.035$. The scattering length for the applied magnetic field $B=910 \mathrm{G}$ is $a_{F}=-0.38 \mu \mathrm{m}=-0.14 a_{z}$ [30], which corresponds to $y=-0.16$ at the droplet center. Here $a_{z}$ $=\left[\hbar /\left(m \omega_{z}\right)\right]^{1 / 2}$. Figure 1(a) compares the observed full width half maximum (FWHM) of the transverse and axial size of the expanding cloud as a function of time [1] with the ones obtained by the numerical integration of the TDNLSE, based on the MC and EBCS equation of state. The TDNLSE is solved numerically by using a finite-difference algorithm [31] on a real-space grid.

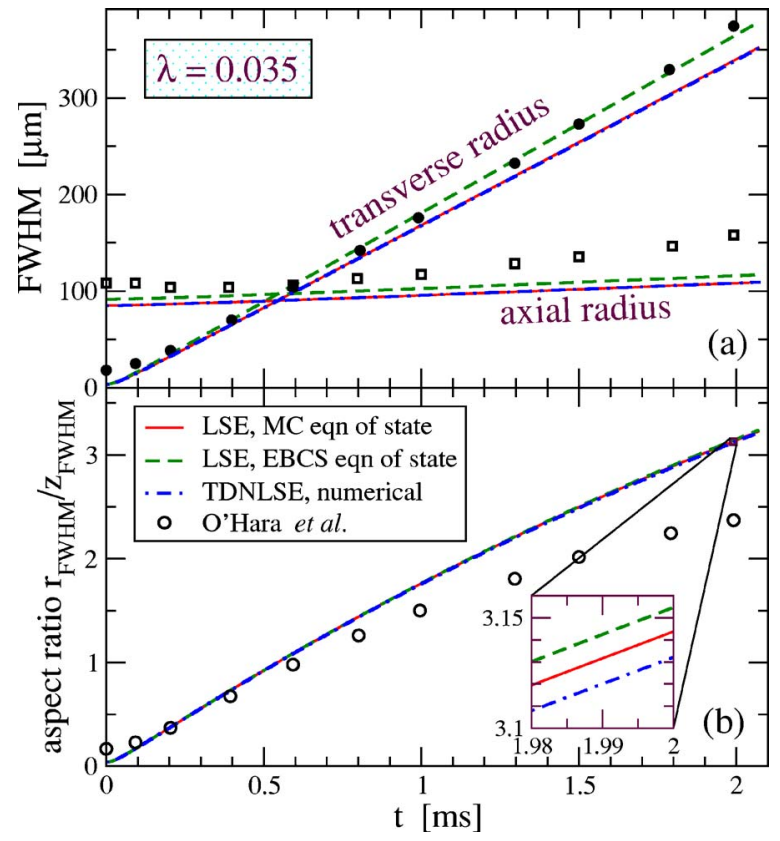

FIG. 1. (Color online) Expansion of a cloud of $1.5 \times 10^{5}{ }^{6} \mathrm{Li}$ atoms released from a trap as realized in Ref. [1], with anisotropy $\lambda=\omega_{z} / \omega_{\rho}=0.035$. (a) Transverse and axial radii of the ${ }^{6} \mathrm{Li}$ atomic cloud close to the unitarity limit: $a_{F}=-0.14 a_{z}$ which corresponds to $y=-0.16$. (b) Aspect ratio of the cloud as function of the time $t$. Circles and squares: experimental data of Ref. [1]; dot-dashed lines: TDNLSE with the MC equation of state; solid lines: LSE with the MC equation of state; dashed lines: LSE with the EBCS equation of state.

Figure 1(a) shows that the expanding gas accelerates more strongly in the radial direction, where the confinement is tighter than axially. Accordingly, the cloud undergoes a shape transition: from a cigar to disk. This is a consequence of superfluidity and interaction: a noninteracting or a normal Fermi gas would undergo a ballistic expansion, leading eventually to a spherical shape [8]. In Fig. 1 the TDNLSE results are plotted as dot-dashed lines and show a fair agreement with the experimental data. It is important to observe that the present theory does not rely on any fitting parameters, while the model curves shown in Ref. [1] critically depend on the choice of the initial widths. The initial profile for the TDNLSE is obtained by running the code integrating Eq. (6) in imaginary time until the confined ground state is filtered out. Figure 1(b) plots the droplet aspect ratio showing that the theory overestimates the experimental data.

From the TDNLSE one can deduce Landau's hydrodynamic equations of superfluids at zero temperature, by setting $\psi(\mathbf{r}, t)=\sqrt{n(\mathbf{r}, t)} e^{i S(\mathbf{r}, t)}$ and $\mathbf{v}(\mathbf{r}, t)=(\hbar / m) \nabla S(\mathbf{r}, t)$, and neglecting the quantum-pressure term $\left(-\hbar^{2} \nabla^{2} \sqrt{n}\right) /(2 m \sqrt{n})$, that is expected to be comparably small for a large number $N$ of particles $[16,17,29]$. These hydrodynamic equations are

$$
\begin{gathered}
\partial_{t} n+\boldsymbol{\nabla} \cdot(n \mathbf{v})=0, \\
m \partial_{t} \mathbf{v}+\boldsymbol{\nabla}\left[\mu(n)+U(\mathbf{r}, t)+\frac{1}{2} m v^{2}\right]=0 .
\end{gathered}
$$

In this approximation, the stationary state in the trap is given by the Thomas-Fermi profile $n_{0}(\mathbf{r})=\mu^{-1}[\bar{\mu}-U(\mathbf{r}, 0)]$. Here 
$\bar{\mu}$, the chemical potential of the inhomogeneous system, is fixed by the normalization condition $N=\int d^{3} \mathbf{r} n_{0}(\mathbf{r})$. We impose that the hydrodynamic equations satisfy the scaling solutions $n(\mathbf{r}, t)=n_{0}\left(x / b_{1}(t), y / b_{2}(t), z / b_{3}(t)\right) / \bar{b}(t)$ and $\mathbf{v}(\mathbf{r}, t)$ $=\left(x \dot{b}_{1}(t) / b_{1}(t), y \dot{b}_{2}(t) / b_{2}(t), z \dot{b}_{3}(t) / b_{3}(t)\right), \quad$ where $\bar{b}(t)$ $=\Pi_{k=1}^{3} b_{k}(t)$. We obtain three differential equations for the scaling variables $b_{j}(t)$, with $j=1,2,3=\rho, \rho, z$. These scaling differential equations depend also on the space vector $\mathbf{r}$. Only if the chemical potential satisfies a polytropic power law $\mu(n)=C n^{\gamma}$ then the space dependence drops out $[14,16]$. In our problem $\mu(n)$ is not a power law but we expect that the dynamics can be well approximated by evaluating the scaling differential equations at the center $(\mathbf{r}=\mathbf{0})$ of the cloud [32]. In this case the variables $b_{j}(t)$ satisfy the local scaling equations (LSE)

$$
\ddot{b}_{j}(t)+\bar{\omega}_{j}(t)^{2} b_{j}(t)=\frac{\omega_{j}^{2}}{\bar{b}(t)} \frac{(\partial \mu / \partial n)\left[n_{0}(\mathbf{0}) / \bar{b}(t)\right]}{(\partial \mu / \partial n)\left[n_{0}(\mathbf{0})\right]} .
$$

The coupled ordinary differential equations (12) are integrated accurately and efficiently to arbitrary time by standard algorithms. We check the reliability of the LSE approach by comparing their numerical solutions to the expansion obtained by using the full TDNLSE (6), both within the MC equation of state (7). Figure 1 reports the LSE results as solid lines, clearly showing that the LSE are extremely accurate: solid lines are practically superimposed to dotted-dashed lines [relative difference $\ll 1 \%$, see the inset of Fig. 1(b)]. Figure 1(a) also reports the transverse and axial radii obtained by solving the LSE with the chemical potential $\mu(n)$ given by the EBCS equations (8) and (9). Remarkably the mean-field EBCS results are closer to the experimental data than the MC results. The two theories essentially coincide for the aspect ratio.

In Ref. [4] the free expansion of $7 \times 10^{4}$ cold ${ }^{6} \mathrm{Li}$ atoms was studied for different values of $y=\left(k_{F} a_{F}\right)^{-1}$ around the Feshbach resonance $(y=0)$. Unfortunately, in this experiment the thermal component is not negligible and thus the comparison with the present $T=0$ theory is not fully satisfactory. Figure 2 compares the experimental data of Ref. [4] with the LSE based on both the MC and EBCS equations of state. Figure 2 shows that the aspect ratio predicted by the two $T$ $=0$ theories exceeds the finite-temperature experimental results. This is not surprising because the thermal component tends to suppress the hydrodynamic expansion of the superfluid. On the other hand, the released energy of the atomic gas is well described by the two $T=0$ theories, and again the mean-field theory seems more accurate, also probably due to the thermal component. For completeness, Fig. 2(c) reports the actual released energy $\int d^{3} \mathbf{r} n_{0}(\mathbf{r}) \mathcal{E}\left(n_{0}(\mathbf{r})\right)$.

In the two experiments of Refs. [1,4] the time evolution is sufficiently short for a full TDNLSE simulation. It would be computationally impractical to integrate the TDNLSE for times much longer than $\omega_{H}^{-1}$, where $\omega_{H}=\left(\omega_{\rho}^{2} \omega_{z}\right)^{1 / 3}$. As the LSE are very reliable at small and intermediate times, we use them to investigate the time evolution of the Fermi cloud for longer times. Figure 3 shows the aspect ratio of the expanding cloud as a function of the inverse interaction parameter $y$

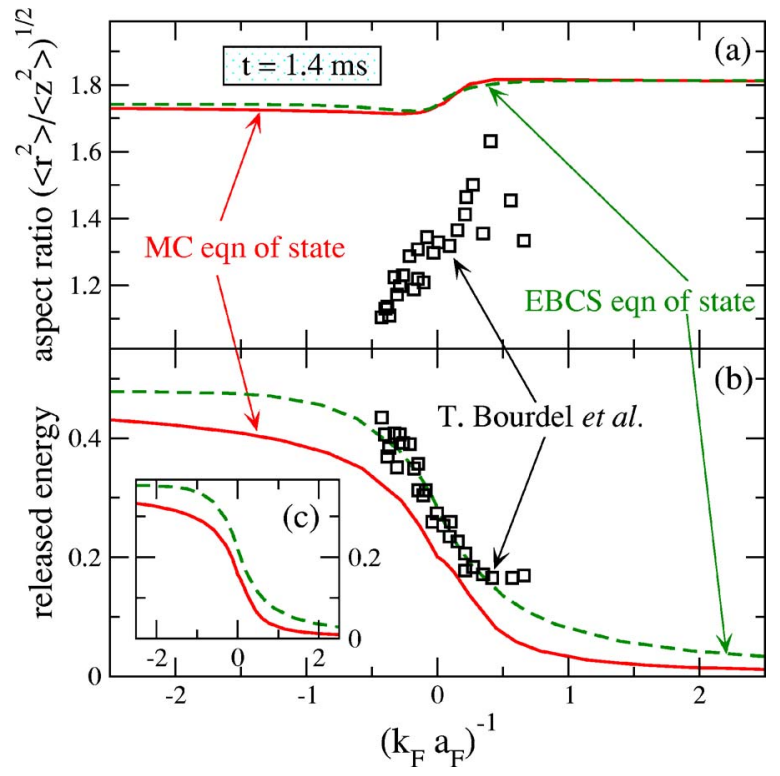

FIG. 2. (Color online) Properties of a ${ }^{6} \mathrm{Li}$ cloud after $1.4 \mathrm{~ms}$ expansion from the trap realized in Ref. [4], of anisotropy $\lambda$ $=\omega_{z} / \omega_{\rho}=0.34$. (a) Aspect ratio of the ${ }^{6} \mathrm{Li}$ atomic cloud as a function of the inverse interaction parameter $y=\left(k_{F} a_{F}\right)^{-1}$. (b) Released energy of the same cloud defined as in Ref. [4] based on the rms widths of the cloud. (c) Actual released energy of the atomic cloud. Squares report the experimental data of Ref. [4]; solid lines: LSE with the MC equation of state; dashed lines: LSE with the EBCS equation of state.

at subsequent time intervals. At $t=0$ the aspect ratio equals the trap anisotropy $\lambda=0.34$. According to these calculations, during the cloud expansion the aspect ratio in the BCS regime $(y \ll-1)$ is measurably different from the one of the $\mathrm{BEC}$ regime $(y \gg 1)$. Thus the free expansion enables one to

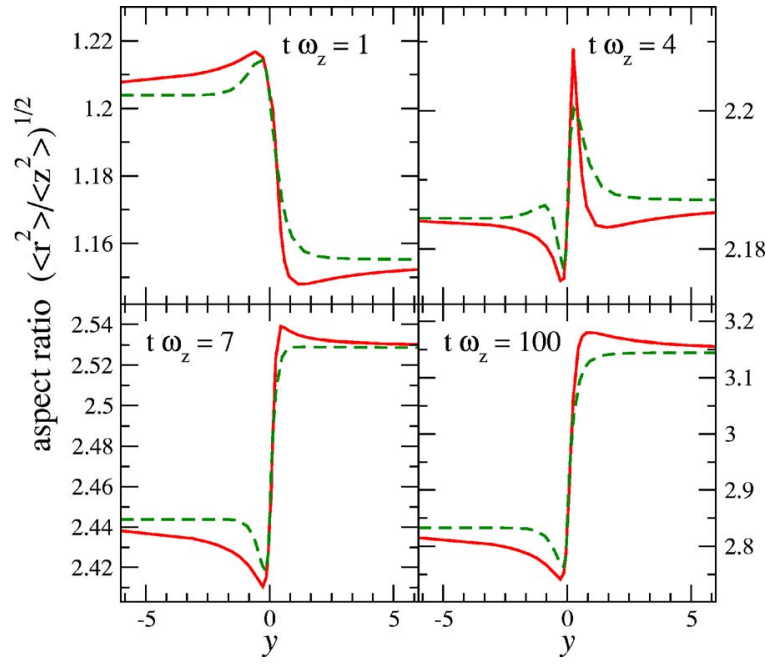

FIG. 3. (Color online) Successive frames of the aspect ratio of the Fermi gas as a function of the inverse interaction parameter $y$ $=\left(k_{F} a_{F}\right)^{-1}$ in the experimental conditions of Ref. [4]. At $t=0$ the Fermi cloud is cigar-shaped with a constant aspect ratio equal to the initial trap anisotropy $\lambda=\omega_{z} / \omega_{\rho}=0.34$. Solid lines: LSE with the MC equation of state; dashed lines: LSE with the EBCS equation of state. 
recognize the regime involved. Figure 3 predicts a novel interesting effect: initially $\left(t \omega_{H} \lesssim 3\right)$ the cloud aspect ratio evolves faster in the BCS region, but then at some intermediate time (here $t \omega_{H} \simeq 4$ ) the BEC side reaches and eventually overtakes the BCS side at larger times (here $t \omega_{H} \geqslant 5$ ). Of course, the detailed sequence of deformations depends on the experimental conditions and in particular on the initial anisotropy, but the qualitative trend of an initially faster reversal on the BCS side, later surpassed by the BEC gas, is predicted for the expansion of any initially cigar-shaped interacting fermionic cloud. Similarly, starting from a disk-shaped cloud $(\lambda>1)$, the aspect ratio reduces more quickly initially on the $\mathrm{BCS}$ side, and later on the BEC side.

\section{DISCUSSION}

Comparison of the EBCS (dashed lines) and MC (solid lines) data shows that beyond mean-field effects do not alter qualitatively the general trend, but they affect the aspect ratio quantitatively, to an extent which could be appreciated by very accurate experiments carried out at extremely low temperature. In particular, the mean-field curves flatten to the asymptotic values (for $|y| \gg 1$ ) closer to the unitary limit than the MC ones. Present-day experimental data, including measurements of collective oscillation frequencies [9,10,12-17], are equally well compatible with the EBCS mean field and the MC-based analysis accounting for beyond mean-field effects. New experiments could shed light on these correlation effects and verify the predictions of the present calculations.

\section{ACKNOWLEDGMENT}

This work was funded in part by the EU's 6th Framework Programme through the NANOQUANTA Network of Excellence (Grant No. NMP4-CT-2004-500198).
[1] K. M. O'Hara, S. L. Hemmer, M. E. Gehm, S. R. Granade, and J. E. Thomas, Science 298, 2179 (2002).

[2] M. Greiner, C. A. Regal, and D. S. Jin, Nature (London) 426, 537 (2003).

[3] S. Jochim et al., Science 302, 2101 (2003).

[4] T. Bourdel et al., Phys. Rev. Lett. 93, 050401 (2004).

[5] A. J. Leggett, in Modern Trends in the Theory of Condensed Matter, edited by A. Pekalski and R. Przystawa (SpringerVerlag, Berlin, 1980).

[6] P. Nozières and S. Schmitt-Rink, J. Low Temp. Phys. 59, 195 (1985).

[7] J. R. Engelbrecht, M. Randeria, and C. A. R. Sa de Melo, Phys. Rev. B 55, 15153 (1997).

[8] L. Pitaevskii and S. Stringari, Science 298, 2144 (2002); C. Menotti, P. Pedri, and S. Stringari, Phys. Rev. Lett. 89, 250402 (2002).

[9] J. Kinast, S. L. Hemmer, M. E. Gehm, A. Turlapov, and J. E. Thomas, Phys. Rev. Lett. 92, 150402 (2004); J. Kinast, A. Turlapov, and J. E. Thomas, Phys. Rev. A 70, 051401(R) (2004).

[10] M. Bartenstein et al., Phys. Rev. Lett. 92, 203201 (2004).

[11] A. Perali, P. Pieri, L. Pisani, and G. C. Strinati, Phys. Rev. Lett. 92, 220404 (2004); A. Perali, P. Pieri, and G. C. Strinati, ibid. 93, 100404 (2004).

[12] S. Stringari, Europhys. Lett. 65, 749 (2004).

[13] R. Combescot and X. Leyronas, Phys. Rev. Lett. 93, 138901 (2004).

[14] H. Hu, A. Minguzzi, X. J. Liu, and M. P. Tosi, Phys. Rev. Lett. 93, 190403 (2004).

[15] H. Heiselberg, Phys. Rev. Lett. 93, 040402 (2004).

[16] Y. E. Kim and A. L. Zubarev, Phys. Rev. A 70, 033612
(2004); Phys. Lett. A 397, 327 (2004); J. Phys. B 38, L243 (2005).

[17] N. Manini and L. Salasnich, Phys. Rev. A 71, 033625 (2005).

[18] M. W. Zwierlein et al., Phys. Rev. Lett. 91, 250401 (2003).

[19] G. Ortiz and J. Dukelsky, Phys. Rev. A 72, 043611 (2005).

[20] L. Salasnich, N. Manini, and A. Parola, Phys. Rev. A 72, 023621 (2005).

[21] G. E. Astrakharchik, J. Boronat, J. Casulleras, and S. Giorgini, Phys. Rev. Lett. 95, 230405 (2005).

[22] A. Bulgac and Y. Yu, Phys. Rev. Lett. 91, 190404 (2003).

[23] M. W. Zwierlein, J. R. Abo-Shaeer, A. Schirotzek, C. H. Schunck, and W. Ketterle, Nature (London) 435, 1047 (2005).

[24] G. A. Baker, Jr., Phys. Rev. C 60, 054311 (1999); H. Heiselberg, Phys. Rev. A 63, 043606 (2001).

[25] D. S. Petrov, C. Salomon, and G. V. Shlyapnikov, Phys. Rev. Lett. 93, 090404 (2004).

[26] G. E. Astrakharchik, J. Boronat, J. Casulleras, and S. Giorgini, Phys. Rev. Lett. 93, 200404 (2004).

[27] J. Carlson, S.-Y. Chang, V. R. Pandharipande, and K. E. Schmidt, Phys. Rev. Lett. 91, 050401 (2003); S.-Y. Chang, V. R. Pandharipande, J. Carlson, and K. E. Schmidt, Phys. Rev. A 70, 043602 (2004).

[28] M. Marini, F. Pistolesi, and G. C. Strinati, Eur. Phys. J. B 1, 151 (1998).

[29] E. Taylor and A. Griffin, Phys. Rev. A 72, 053630 (2005).

[30] M. Bartenstein et al., Phys. Rev. Lett. 94, 103201 (2005).

[31] E. Cerboneschi, R. Mannella, R. Arimondo, and L. Salasnich, Phys. Lett. A 249, 495 (1998).

[32] L. Salasnich, A. Parola, and L. Reatto, Phys. Rev. A 72, 025602 (2005). 